\title{
A Machine Vision System for Detecting Fertile Eggs in the Incubation Industry
}

\author{
Mahdi Hashemzadeh*, Nacer Farajzadeh \\ Faculty of Information Technology and Computer Engineering, \\ Azarbaijan Shahid Madani University, Tabriz, Iran \\ E-mail: hashemzadeh@azaruniv.edu \\ n.farajzadeh@azaruniv.edu
}

Received 19 August 2015

Accepted 8 May 2016

\begin{abstract}
One of the important factors in increasing the productivity of the incubation industry is to be sure that the eggs placed in the incubators are fertile. In this research, a fertility detection machine vision system is developed and evaluated. To this end, a mechatronic machine is fabricated for acquiring accurate digital images of eggs without harming them. An appropriate and cheap light source is also introduced for illuminating the eggs, which potentially enables a CCD camera to obtain good quality and informative images from inner side of the eggs. Finally, a robust machine vision algorithm is developed to process the captured images and distinguish fertile eggs from infertile ones. In order to evaluate the system, a large egg image dataset is provided using 240 incubated eggs (including 190 fertile and 50 infertile eggs). The fertility detection accuracy of the system on the provided dataset reaches $47.13 \%$ at day 1 of incubation, $81.41 \%$ at day $2,93.08 \%$ at day $3,97.73 \%$ at day 4 , and $98.25 \%$ at day 5. Comparisons with existing approaches show that the proposed method achieves a superior performance. The obtained results indicate that the proposed system is highly reliable and applicable in the incubation industry.
\end{abstract}

Keywords: Machine Vision, Image Processing, Egg, Fertility Detection, Incubation Industry, AutoCandling.

\section{Introduction}

Incubation industry is one of the main industries in the food supplement chain and plays a great role in raising avian ${ }^{1}$. Hatchability of eggs is an important factor in incubation industry. It is influenced by many factors such as egg handling, fertility of eggs, breeder flock problem, etc. However, the most critical factor is to be sure that the eggs placed in the incubator are truly fertile eggs ${ }^{2}$. Early detection of infertile and non-hatchable eggs would benefits hatcheries and poultry breeding farms by saving space, handling costs, and preventing contamination from exploder eggs ${ }^{2}$. In most incubation centers, the process of separating fertilized and non-fertilized eggs is performed by experts (through human vision and traditional ways) before loading the eggs into the incubators. After loading the eggs into the incubators, some experts periodically check them to make sure that they are truly fertilized. Sometimes, candling machines are used in this process. In these machines, eggs are exposed to a sharp dim of light. Hence, the inner side of the eggs can be visible and one can decide whether there exists an embryo inside them or not. If the inside of an egg is totally clear without any stains, it means that it is not fer-

\footnotetext{
* Corresponding author.
} 
tile; but if there is a black spot alongside with some blood veins (spider like), it is considered as a fertile $\mathrm{egg}^{3}$.

The candling process is labor consuming, and is not very efficient due to exhaustion and sight mistakes of workers who have to check thousands of eggs per day. Therefore, usually only some eggs are randomly selected for determination of flock fertility, which means most infertile eggs will remain in the incubator ${ }^{2}$. Moreover to the capacity issue, the remaining infertile eggs inside the incubator will be contaminated, and, as a consequence, some gases will be released into the environment making the situation problematic for the other healthy embryos ${ }^{1}$. Therefore, the development of an accurate, rapid and cheap machine vision system for detecting the infertile eggs in appropriate time would be advantageous to the incubation industry.

To date, various works have been done based on machine vision techniques to perform some tasks in this industry. Some of the research is dedicated to the detection of cracks and dirt on eggs, $4,5,6,7,8,9,10,11,12,13$ or measuring physical coordinates of eggs $5,11,14,15,16,17$. In a similar manner to the candling machines, most of these researches usually use light sources such as the incandescent lamp, fluorescent lamp or halogen lamp to back-illuminate the egg. The candled eggs are imaged, and the captured images are processed to extract some physical features from the eggshells' surface. The extracted features are used to perform the target inspections. Although, the above mentioned light sources are suitable to provide necessary illumination for detection of defects on the eggshell surface from the captured images, the acquired images lack the sufficient quality to determine the fertility of eggs. In other words, the conventional light sources used in the candling machines and the previous works are not appropriate enough to reveal the details of the inner side of eggs such as embryo and its blood veins.

Some researchers have tried to determine the fertility of eggs by employing equipment such as MRI, infrared hyperspectral imaging, or thermal cameras $^{2,18,19,20,21}$. However, using this expensive equipment would not be economical, especially in the case of mass production. Additionally, possi- ble radiation produced from such equipment may harm the embryos. While considering the illumination power of the light source, we should also take into account its power consumption and its coolness. Considering the physical and the chemical structure of an egg, heat can change the content inside of it and damage its embryo. Usually, embryos cannot tolerate a heat increase of more than $2{ }^{\circ} \mathrm{C}^{1}$.

In this paper, we propose an automatic fertility detection system based on digital images acquired by using an appropriate light source and a conventional CCD camera. The proposed system is not only cheap, but also guarantees the safety of the eggs. In brief, the contributions of this research are threefold:

(i) Designing and implementing a mechatronic auto-candling machine that can provide digital images of eggs with a high accuracy and no harm.

(ii) Introducing a very cheap light source which potentially enables a CCD camera to obtain a good quality image from inner side of the eggs. This light source does not harm the embryos in any way.

(iii) Developing a machine vision algorithm for efficiently detecting the fertility of hatching eggs with a high accuracy.

In the conducted experiments, the fertility detection accuracy is $47.13 \%$ at day 1 of incubation, $81.41 \%$ at day $2,93.08 \%$ at day $3,97.73 \%$ at day 4, and $98.25 \%$ at day 5. Comparisons with existing approaches show that the proposed method outperforms more complex competitors. The obtained results also indicate that the proposed system is highly reliable and applicable in incubation industry.

The rest of this paper is organized as follows. Related work is reviewed in next section. Section 3 describes the details of the proposed system. Our experimental results are presented and discussed in Section 4. Finally, in Section 5, we draw conclusions and discuss possible directions for future work. 


\section{Related Work}

In this section, we briefly review some of the related research conducted in the literature. As one of the early works, Patel et al. ${ }^{4}$ developed a system to inspect cracks, blood stains and dirt on the eggshell surface. They used a 25 Watt incandescent candling lamp to back-illuminate the egg and then took its image. Subsequently, histograms from color images were used to conduct highly accurate sorting tests. Fang et al. ${ }^{9}$ implemented a machine vision system to detect cracks on the eggs. In this system, a circular fluorescent lamp was used as a light source for imaging. In another work, Ibrahim et al. ${ }^{11}$ used a light source including two incandescent lamps with yellow light which was attached beside the camera to illuminate the eggs. The obtained images were processed and the edges of dirt on the surface of the eggs were detected.

Lawrence et al. ${ }^{6}$ developed a machine vision system assembled with pressure chamber to detect micro cracks in the eggshell. A small chamber was built to create vacuum and force to open the cracks on a given egg, and then digital images of the egg were taken. The system was designed for inspecting a single egg at a time. Later, they reported a modified pressure system to detect micro cracks in 15 eggs at a time ${ }^{7}$. Javadikia et al. ${ }^{16}$ tried to measure the width and the length of a given egg by real time image processing techniques.

Some works based on machine vision techniques have been also developed to detect fertility and embryo development. Das et al. ${ }^{22,23}$ implemented a system to identify fertile hatching eggs by using image histogram as a feature and an artificial neural network as a classifier. Images of eggs were acquired using backlighting with a high intensity candling lamp. The gray level histograms of the images were enhanced and used as inputs to a neural network.

Zhu et al. ${ }^{24}$ proposed a method based on machine vision and least square support vector machine (LS-SVM) for identification of fertile and infertile eggs. A single 150 Watt, 24 Volt-DC tungsten-halogen lamp was used to back-illuminate the egg. Digital images were captured by a highresolution digital camera, and features such as egg shape index, roundness, elongation, geometric moment along with the color mean information of the egg yolk region were extracted. LS-SVM algorithm was used to establish a classification model to separate the fertile eggs from infertile ones.

Bamelis et al. ${ }^{18}$ utilized the spectrophotometric method to determine embryo development of eggs during the first 12 days of incubation. They concluded that the embryo development can be detected using light transmission from 108 hours (day 4.5) of incubation. Lin et al. ${ }^{21}$ proposed a thermal imaging system for the identification and filtering of fertile eggs. Based on the thermal information and a fuzzy system, they could obtain a proper value for a threshold which was used to judge as to whether a given egg is fertile or not.

Some researchers have tried to utilize the advanced hyperspectral imaging technique to detect egg fertility and embryo development ${ }^{2,19,20}$. A hyperspectral image contains not only spatial information, but also spectral information for each pixel in the image. Lawrence et al. ${ }^{19}$ applied this technique for detecting early embryo development of hatching eggs at the first three days of incubation. They claimed that the embryo classification results were about $96 \%$ at day 0 of incubation, $92 \%$ at day 1 , and $100 \%$ at days 2 and 3 . The same research group later designed a system to detect the fertility of hatching eggs by utilizing the hyperspectral imaging technique ${ }^{20}$. However, the prediction model produced much lower classification results $(71 \%$ for day $0,63 \%$ for day $1,65 \%$ for day 2 , and $83 \%$ for day 3). Liu et al. ${ }^{2}$ developed a near-infrared hyperspectral imaging system to detect fertility and early embryo development of eggs. They measured some spectral transmission features of hyperspectral images and used them to detect the fertility. The classification rates were $78.8 \%$ at day $1,74.1 \%$ at day 2 , $81.8 \%$ at day 3 , and $84.1 \%$ at day 4 of incubation.

However, as mentioned in Introduction, using these expensive equipment would not be economical and practical in the case of mass production. In this work, we develop a cheap and economical machine vision system for detecting the fertility of hatching eggs with a better accuracy. 


\section{Proposed System}

The proposed system consists of three major phases: (1) designing and implementation of a mechatronic auto-candling machine, (2) building the imaging light source, and (3) fertility detection. The details of all these phases are provided in the following subsections.

\subsection{Auto-candling machine}

Considering the sensitivity and the importance of an accurate digital imaging of eggs, a mechatronic auto-candling machine for taking images of the eggs is designed and implemented. The proposed machine gives us the opportunity for high accuracy imagining with different setting options while guaranteeing the safety of the eggs. Figure 1 shows a laboratory size of the proposed candling machine. This machine consists of three parts: a mechanical part, an imaging light source, and an electronic part.

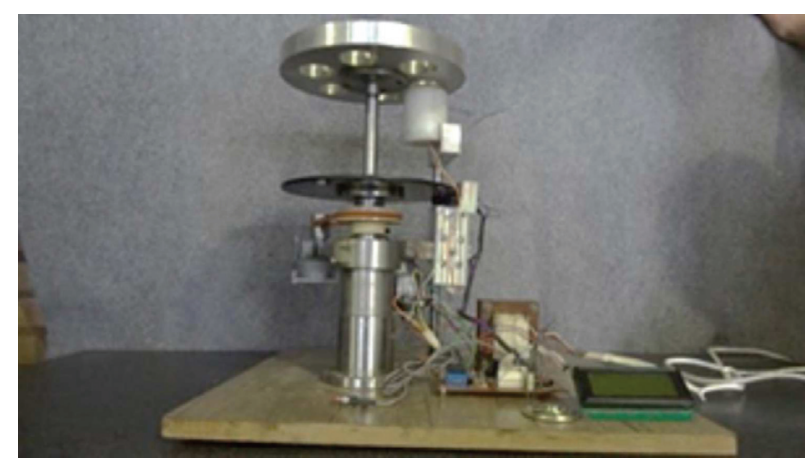

Fig. 1. Auto-candling machine in a laboratory size.

The mechanical part includes an engine that moves a platform which has rooms for six eggs. The light source is positioned under the platform for illuminating the egg. The eggs are placed in order on the light source. Then, the light source automatically starts working and the imaging process is done by a camera that is fixed next to the eggs. We should note that the imaging is performed in a totally dark environment. Four images from four different sides (angles) of each egg are taken and sent to the fertility detection program that is run in a computer. After imaging, the engine turns the platform, and the next egg goes under the same process.

The electronic part controls the engine and automatic on/off switching of the light source. This part includes a microcontroller, power supply, control relays, optical counters and a display screen. In addition to a fine tuning process required for accurate imaging, tools for counting the eggs and adjusting the light intensity for different types of eggs (e.g. with different thickness of the eggshell) are provided by the electronic part as well. In the current version of implemented machine, the light intensity is manually adjusted using a potentiometer. Also, the distance between the eggs and the light source is adjusted by a screw next to the light source holder. However, in order to fully automate the system, image-based feedback controls can be used to accurately perform these adjustments.

\subsection{Imaging light source}

Choosing an appropriate light source to illuminate the eggs is a key part of the imaging process. That is, the more visibility in seeing the inner side of an egg, the higher the accuracy in the digital imaging. To choose the type of the light source, we consulted with physics and optic experts and also conducted some empirical tests, some of which are explained in Subsection 4.6. By evaluating the results of our experiments, we decided to use an LED (Light Emitting Diode) with a white light and linear radiation as our light source. Some important characteristics of this light source are listed in Table 1.

Table 1. Important characteristics of the LED light source used in the proposed machine.

\begin{tabular}{lccl}
\hline Property & Symbol & Value & Unit \\
\hline Light temperature & $C C T$ & 6500 & Kelvin \\
Luminous & $\Phi v$ & 30 & Lumen \\
Power & $W$ & 0.12 & Watt \\
Maximum forward current & $I_{F}$ & 50 & Milli Amp \\
Reverse Voltage & $V_{R}$ & 5 & Volt \\
\hline
\end{tabular}

The LED light is placed in a cylinder box (a tube) with an appropriate radiation angle and is installed under the egg holding platform in the auto-candling machine. Figure 2 demonstrates the lighting box when the light source is turned on. The position of 
the light source fixed on the machine is also visible in Fig. 1. This light source provides the necessary illumination to clearly image the details of the inner side of an egg. Figure 3 shows a sample egg image taken by using this light source. As shown in this image, the embryo and its blood veins are clearly visible inside the egg.

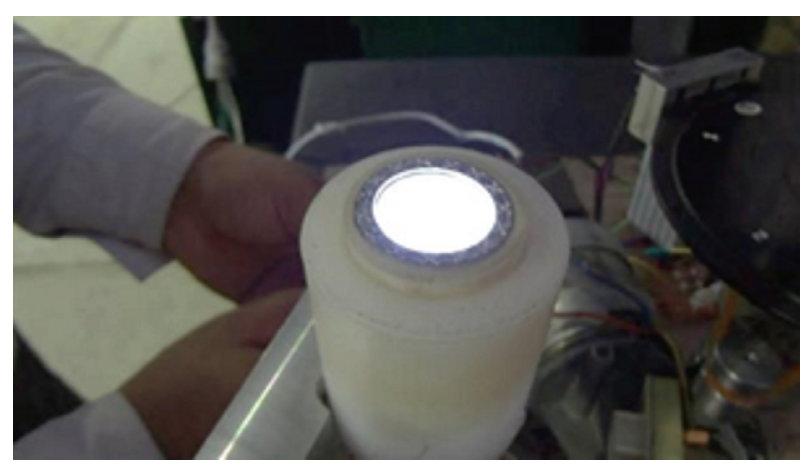

Fig. 2. A close view of the light source.

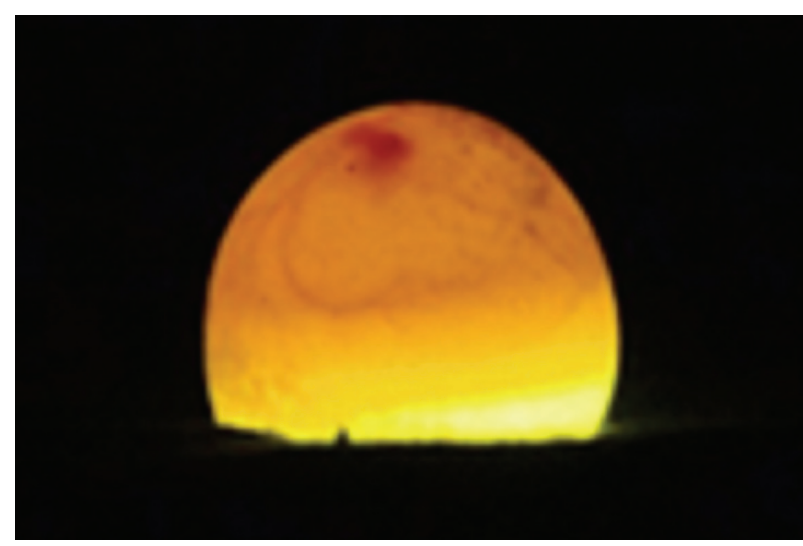

Fig. 3. A sample digital image taken by the proposed candling-machine.

\subsection{Fertility detection}

The captured images are processed by fertility detection process. This process aims to detect the part of an image that shows the embryo and confirm the fertility of a given egg with a high accuracy. In order to determine the fertility of an egg, all four images taken from an egg are processed separately. If the fertility is detected at least in one of the images, the corresponding egg is classified as a fertile one.

An outline of the proposed fertility detection procedure is illustrated in Fig. 4. The steps in this procedure are divided into two phases: (1) preprocessing, and (2) binarization and classification. The details of each phase are presented in the following subsections.

\subsubsection{Preprocessing}

Region of Interest: the input RGB image is converted to a grayscale image. Then, the region of interest (i.e. the egg) is localized and a sub-image is created to be processed in the next steps. By assuming that the background of egg images is almost black, extraction of sub-image is simply done using the intensity information and applying some morphology operations. In other words, the sub-image is easily obtained by detecting the area whose brightness changes abruptly (i.e. the edges of the eggs).

Histogram Equalization: in order to increase the visibility of embryo inside the egg, we increase the image contrast. To this end, we equalize the histogram of the sub-image by utilizing the contrastlimited adaptive histogram equalization (CLAHE) method proposed in Ref. ${ }^{25}$. Adaptive histogram equalization (AHE) is an image processing technique that is used to improve the contrast in images. It differs from ordinary histogram equalization in the respect that the adaptive method computes several histograms, each corresponding to a distinct section of the image, and uses them to redistribute the lightness values over the image. It is therefore suitable for improving the local contrast and enhancing the definitions of edges in each region of an image. CLAHE, on the other hand, was developed to prevent the over-amplification of noise that adaptive histogram equalization can give rise to 25 . 


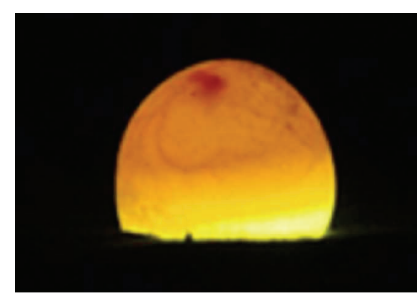

(1) Input RGB image

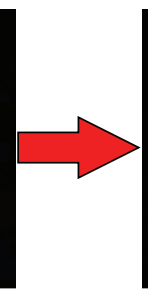

(2)
(2) Converting to grayscale
image

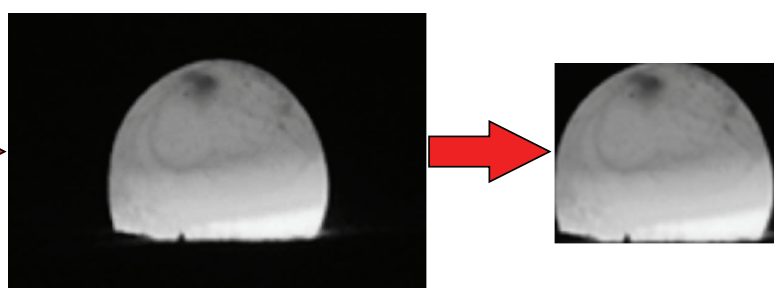

(3) Localizing the egg

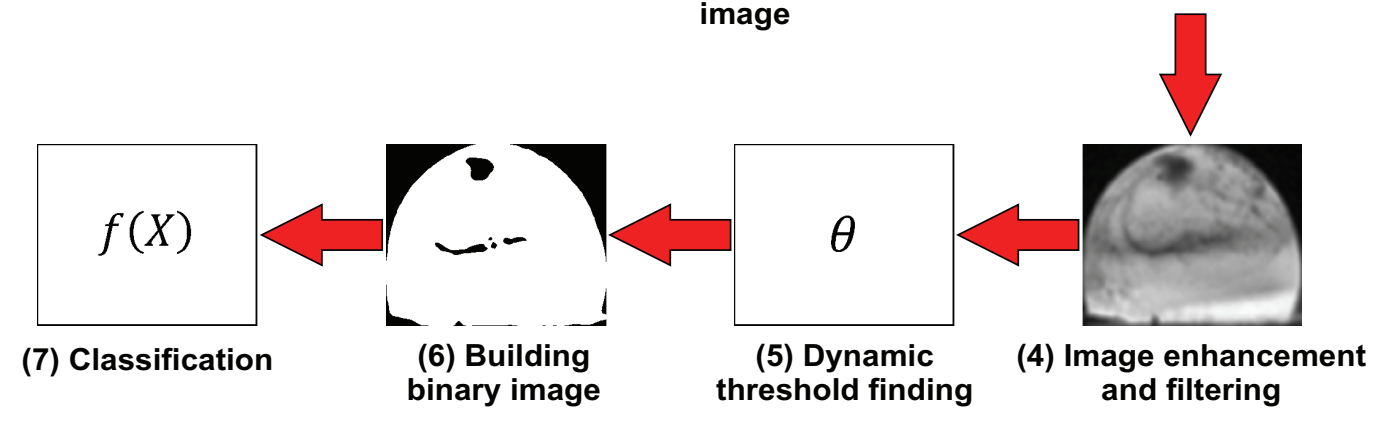

Fig. 4. Outline of the fertility detection process.

Noise removal: to remove possible noises due to use of inexpensive camera or small spots on the eggshell, a median filter with a $3 \times 3$ template and Gaussian smoothing is used to eliminate noises. The median filter is a nonlinear digital filtering technique that is often used to remove noise. Such noise reduction is a typical preprocessing step to improve the results of later processing (for example, edge detection on an image). Median filtering is very widely used in digital image processing because, under certain conditions, it preserves edges while removing noise ${ }^{26}$. A sample of enhanced and filtered subimage is shown in Fig. 4 (step 4).

\subsubsection{Binarization and classification}

We use a thresholding scheme to convert the preprocessed image to a binary image. This is to distinguish pixels of the embryo (dark points) from the other regions (light parts). As different eggs and the embryos differ in some physical properties such as color, size, and blood veins, hence using a fixed threshold value or applying common threshold finding methods such as Otsu algorithm ${ }^{27}$ is not applicable in this system. Therefore, we propose a dynamic threshold finding algorithm to measure a proper threshold value for each individual image. The proposed algorithm is explained in detail in Subsection 3.3.3. By performing the thresholding task, the embryo and its blood veins are indicated with black pixels and the remaining parts are indicated with white pixels. However, the obtained binary image may contain some noise due to spars blood stains or spots on the eggshell surface. The noise is present in the form of small black holes. To remove these holes, a filter with $3 \times 3$ template size is used. A sample filtered binary image is shown in Fig. 4 (step 6).

Finally, the ratio of the black pixels to the white ones inside the egg in the binary image is calculated and used to classify the egg. The classification task is performed based on Eq. (1):

$$
f(x)= \begin{cases}\text { fertile } & x \geqslant \beta \\ \text { infertile } & x<\beta\end{cases}
$$

where $x$ is the ratio of the number of black pixels to the white ones inside the egg in the binary im- 
age, and $\beta$ is a predefined value. We, experimentally, found that choosing the value of $\beta$ equal to $7 \%$ meets the expected accuracy of the system. Different rates close to this value, for example in range of $5 \%$ to $9 \%$, show similar performances. More discussion regarding to choosing a proper value for $\beta$ is presented in Subsection 4.7. However, for a higher efficiency and in order to have a fully automatic machine, one may apply machine learning methods ${ }^{28}$ to build a model for classifying the eggs according to the value of $x$ and the threshold value selected for image binarization task. For this purpose, a method using artificial neural networks is proposed in Subsection 3.3.4.

\subsubsection{Dynamic threshold finding}

Since the color, size, shape and the blood veins of embryos in different eggs are not the same, and also these properties change on different days of incubation, an optimal threshold value should be chosen for binarization of each individual image. To this end, we design a dynamic threshold finding scheme that can automatically find a proper value for the threshold. Algorithm 1 in Fig. 5 presents the detailed procedure of the method. In the conducted experiments (see Subsections 4.2 and 4.5), the threshold values found by the algorithm showed a desired performance.

\subsubsection{Training a neural network to classify the eggs}

In order to have a fully automatic machine, we design an artificial neural network to separate fertile from infertile eggs. That is, instead of using a predefined value of $\beta$ (see subsection 3.3.2) to detect fertility in a binary image, we use a neural network to do the job. To this end, after extracting the binary image, a neural network is fed with two features: (1) the selected threshold value for binarization task and (2) the ratio of the black pixels to the white ones inside the egg in the binary image. Let the selected threshold value for a given image, the ratio of black pixels to white pixels in the corresponding binary image, and the class of the egg are respectively denoted by $\theta, x$, and $c$. A training set is used to train the neural network and a model is induced as in Eq. (2).

$$
c=f(\theta, x)= \begin{cases}1 & \text { fertile } \\ 0 & \text { infertile }\end{cases}
$$

In Eq. (2), $c$ has a binary value which determines if the egg is fertile or not. We use a single hidden layer backpropagation neural network ${ }^{29}$ with 10 neurons in the hidden layer to perform the classification. These neural networks are considered as multilayer networks which are popular in different disciplines (see Ref. ${ }^{29}$ for more descriptions on these types of neural networks). The trained neural network can be used to classify the new input images.

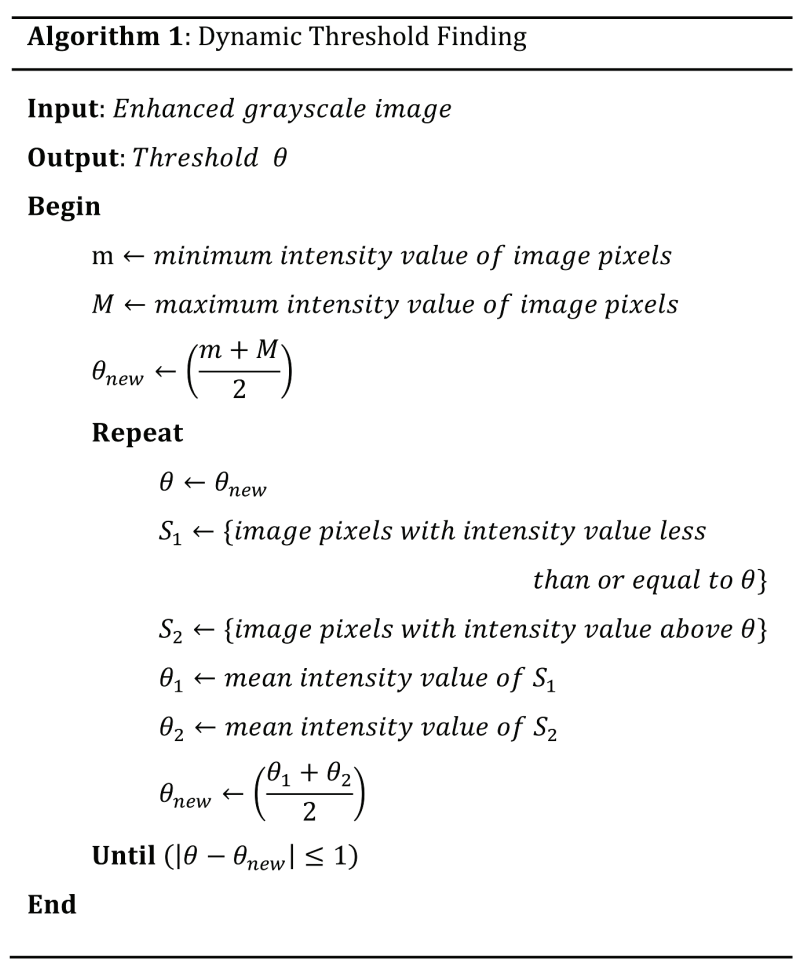

Fig. 5. Dynamic threshold finding algorithm.

\section{Experimental Results}

In this section, we evaluate the performance of the proposed system using a large set of egg images provided for this purpose. Firstly, the preparation process of this dataset is explained in detail. Then, 
the results of our experiments for evaluating the efficiency and the accuracy of the proposed system are discussed and compared with the results of other approaches. Finally, the results of experiments for choosing an appropriate imaging light source are presented.

\subsection{Dataset}

In order to evaluate the fertility detection accuracy of the proposed system on different days of incubation, images of egg samples at different time periods of incubation are required. To this end, we use a laboratory size incubator machine. The capacity of this machine is 48 eggs. Two hundred forty eggs including 190 fertile eggs and 50 infertile eggs are collected from a local hatchery. The process of identification of fertile and infertile eggs is manually performed by some experts. All of the eggs are completely intact without any cracks on the shells. We also apply some incubation preparation on all of the sample eggs such as disinfecting and cleaning dirt and blood on the eggshells. All 240 eggs are loaded into the incubator in six separate sets. Each set contains 40 eggs. The incubation process for each set is performed separately. The first, second, fifth and the sixth sets have 10 infertile eggs and 30 fertile eggs, and the third and the fourth sets have five infertile eggs and 35 fertile ones.

During the incubation, the eggs are candled and imaged every 24 hours for the first five days. Imaging is performed with a Sony Cyber Shot, DSCWX7 CCD camera. The resolution and the distance of imagining are set to $720 \times 480$ pixels and $10 \mathrm{~cm}$ respectively. The captured images are saved in JPEG format. As mentioned in Subsection 3.1, in each attempt, four images from four different sides (angles) are taken. Totally, 4800 (4 images $\times 240$ eggs $\times 5$ days) images are acquired and saved in the dataset. Finally, the eggs are kept in the incubator until the end of day 21 in order to complete the incubation process.

At the end of the incubation process of each set (i.e. after the end of day 21), all the eggs that do not turn in to chickens are kept to be checked by a veterinarian to diagnosis the reason. In the examination results, no fetal injury due to the imaging process or radiation has been reported. This can be considered as a proof of the safety of the proposed auto-candling machine.

\subsection{Experiment 1: Fertility detection results}

All of the images in our dataset are processed by the system and classified into two groups: fertile and infertile. Fertility detection accuracy of six separate hatching sets in five continuous days of the incubation process is shown in Table 2. As this table shows, our systems achieves the average detection accuracy of $93.08 \%$ at day 3 of incubation, $97.73 \%$ at day 4 , and $98.25 \%$ at day 5 . These results indicate that the proposed system is highly reliable and applicable in the incubation industry.

Table 2. Fertility detection accuracy of six sets in five days of incubations.

\begin{tabular}{lccccc}
\hline \multirow{2}{*}{ Set } & \multicolumn{5}{c}{ Day } \\
\cline { 2 - 6 } Set 1 & $48.15 \%$ & $81.48 \%$ & $92.59 \%$ & $96.30 \%$ & $96.30 \%$ \\
Set 2 & $44.00 \%$ & $80.00 \%$ & $96.00 \%$ & $100.0 \%$ & $100.0 \%$ \\
Set 3 & $50.00 \%$ & $78.13 \%$ & $96.63 \%$ & $96.88 \%$ & $100.0 \%$ \\
Set 4 & $41.18 \%$ & $82.35 \%$ & $94.12 \%$ & $97.06 \%$ & $97.06 \%$ \\
Set 5 & $57.14 \%$ & $85.71 \%$ & $92.86 \%$ & $100.0 \%$ & $100.0 \%$ \\
Set 6 & $42.31 \%$ & $80.77 \%$ & $92.31 \%$ & $96.15 \%$ & $96.15 \%$ \\
\hline Average & $\mathbf{4 7 . 1 3 \%}$ & $\mathbf{8 1 . 4 1 \%}$ & $\mathbf{9 3 . 0 8 \%}$ & $\mathbf{9 7 . 7 3 \%}$ & $\mathbf{9 8 . 2 5 \%}$ \\
\hline
\end{tabular}

The image processing and fertility detection phase is implemented in $\mathrm{C}++$ using OpenCV library ${ }^{30,31}$. The procedure is run on a computer with Intel core i7 CPU and 4 GB of RAM. On average, the processing time for each image is 0.22 seconds.

\subsection{Experiment 2: Comparison with other methods}

The performance of the proposed fertility detection machine is compared with results of some other approaches that have quantitatively measured their techniques' performances. Table 3 summarizes the average fertility detection accuracy of different methods during the first five days of incubation. The reported results of methods in Refs.,20,22,23,24 are quoted directly from the related papers. Best rates are bold faced. The "-" mark in some cells of the 
table means that the authors of related paper have not reported any results for the corresponding day of incubation.

From the results in Table 3, it is clear that the higher performances are achieved by the proposed method in most of the incubation days. The detection accuracy of our approach is much better than the approaches proposed in Ref. ${ }^{20}$ and Ref. ${ }^{2}$ which utilized more complex techniques such as hyperspectral imaging system in their methods. The performance of the proposed method is also higher than the methods proposed in Refs. ${ }^{22,23}$ and Ref. ${ }^{24}$ which are also based on the egg images taken by CCD cameras. Although the detection rate of the proposed method on first day of the incubation is lower than the rate of method proposed in Ref. ${ }^{2}$, the results obtained for other days are much better than its results. The effective and appropriate imaging light source that has been utilized in our system and also robust image processing algorithms help our system to achieve more accurate results than the other methods.

Table 3. Fertility detection accuracy of different methods during five days of incubation.

\begin{tabular}{|c|c|c|c|c|c|}
\hline \multirow[b]{2}{*}{ Method } & \multicolumn{5}{|c|}{ Day } \\
\hline & $1 \mathrm{st}$ & 2nd & $3 \mathrm{rd}$ & 4th & 5 th \\
\hline Ours & $47.13 \%$ & $81.41 \%$ & $93.08 \%$ & $97.73 \%$ & $98.25 \%$ \\
\hline Das et al. ${ }^{22,23}$ & - & $67.6 \%$ & $93.5 \%$ & $93.9 \%$ & - \\
\hline Liu et al. ${ }^{2}$ & $78.8 \%$ & $74.1 \%$ & $81.8 \%$ & $84.1 \%$ & - \\
\hline Smith et al. ${ }^{20}$ & $63 \%$ & $65 \%$ & $83 \%$ & - & - \\
\hline Zhu et al. ${ }^{24}$ & - & - & - & - & $92.5 \%$ \\
\hline
\end{tabular}

\subsection{Experiment 3: Evaluating the neural network}

After extracting the binary images and determining the class of the egg in each image, we randomly choose $50 \%$ of the images of each set $(50 \%$ of the images of fertile eggs and $50 \%$ of the images of infertile eggs in each set) to build a training set to train the neural network. Extracted features from the training images (i.e. selected threshold value and the rate of black pixels to white pixels) are served as the inputs of the neural network. The remaining $50 \%$ of the images are used as the test set for evaluating the neural network.

Figure 6 compares the average fertility detection accuracy of the neural network in five days of incubation with the average accuracy of the system without using the neural network (i.e. results presented in Table 2). From this figure, it is clear that the accuracy of the system increases in almost all days when we use the neural network. Obviously, this improvement can be increased if one could use a larger and sufficient training data. As a conclusion, using the neural network as a predicting model not only makes the classification totally automatically, but also it gives us the hope of reaching better results.

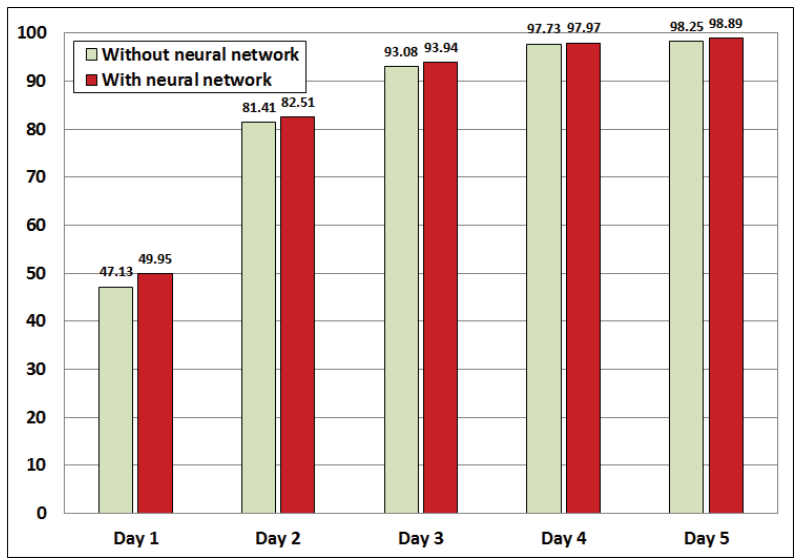

Fig. 6. Comparison of the average fertility detection accuracy (in percentage) in five days of incubation process.

\subsection{Experiment 4: Evaluating the dynamic threshold finding method}

In order to examine the robustness of the proposed dynamic threshold finding method, fertility detection results of the proposed system are compared against the results of equivalent system which uses Otsu method ${ }^{27}$ to find the required threshold value in image binarization step. Otsu algorithm has been widely used in previous related work such as approaches in Refs. ${ }^{10,12,13,17}$. This algorithm exhaustively searches for the threshold that minimizes the intra-class variance defined as a weighted sum of variances of the two classes. For more details about this algorithm see the related paper ${ }^{27}$.

Figure 7 compares the average fertility detection accuracy of two systems in five days of incubation. 
From this figure, it is clear that the system using proposed threshold finding method provides more accurate results than the system utilizing Otsu algorithm. Based on these results, it is obvious that the proposed thresholding scheme has remarkable influence in the performance improvement of the entire system.

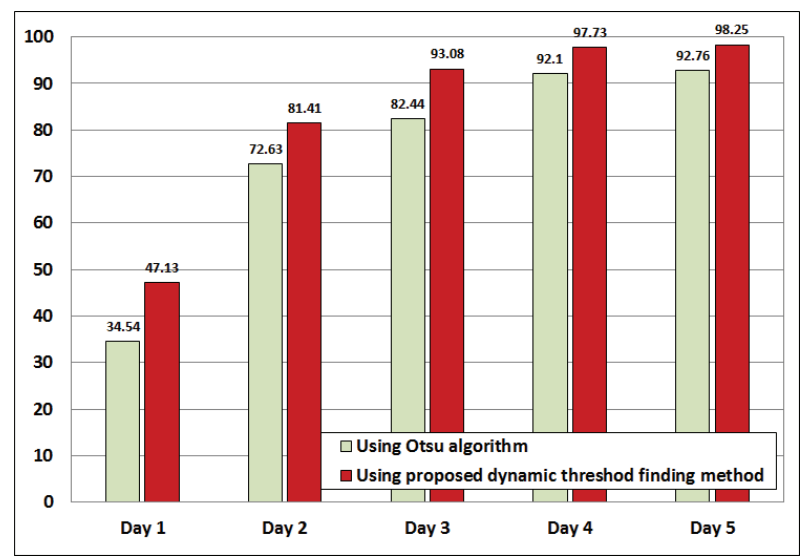

Fig. 7. Comparison of the average fertility detection accuracy (in percentage) of two systems in five days of incubation process.

\subsection{Experiment 5: Empirical tests for choosing an appropriate light source}

In order to choose an appropriate light source for back-illuminating the eggs, different light sources including incandescent lamps, fluorescent lamps, halogen lamps and different LED lights are tested and evaluated. The results of the experiments on each light source are briefly presented in the following.

Incandescent lamps: Incandescent lamps such as 100 Watt and 200 Watt lamps and car headlight lamps are used to illuminate the eggs. Due to using an incandescent filament in these lamps, their light is not absolute white; as a result, the color of the egg turns to yellow and we cannot see any signs of the embryo and blood veins in it. Also, the heat of these lamps is very high which can be harmful for embryos.
Fluorescent lamps: Using fluorescent lamps solves the heat problem but the yellowness of the eggs grows more. Filters with different colors, like red, blue, green and yellow, are tested to solve this problem but it did not work either.

Halogen lamps: The size of the halogen lamps is small in comparison to the incandescent lamps, and they consume low electrical power and produce less heat. With all of these, they increase the yellowness of the eggs, so we cannot see the inner side of the eggs.

LED lights: First we test an LED light with high electrical power (10 Watt). Due to the high light intensity of this LED, the color of the egg changes to red, and we cannot see its inside. Then, we use an LED light with low power (0.12 Watt), which has a quite white and cool light. With this type of LED, we can see very clear details of the inner side of the eggs and can image them clearly. The important characteristics of this light source are mentioned in Subsection 3.2. Figure 8 shows sample images taken by different light sources. As we can see, in the image taken by the low power LED, the inside of the egg is clearly visible.

\subsection{Parameter setting}

The only parameter that we need to set in the fertility detection phase of our approach is the value of threshold $\beta$ (see Eq. (1)). The optimal value of this threshold is determined with respect to the highest average performance of the system on five days of incubation process. Figure 9 shows the average fertility detection accuracies on five days of incubation regarding to the different values of $\beta$. As shown in this figure, choosing the value of $\beta$ equal to $7 \%$ achieves the best accuracy, and the other rates close to this value also show similar performances. On the other hand, setting the value of $\beta$ less than 5\% or higher than $9 \%$ decreases the performance of the system. This is due to false positive detections in the case of lower values, and false negative detections in 
the case of higher values.

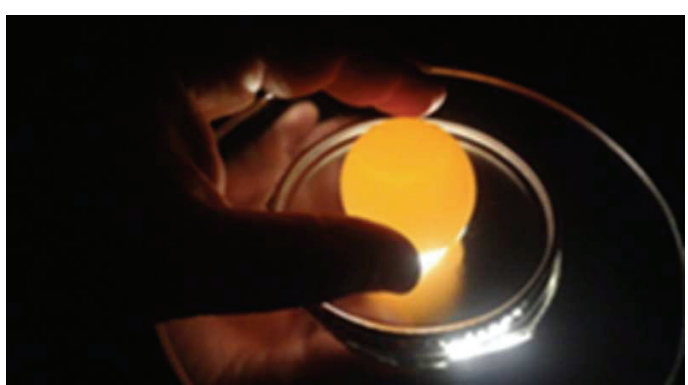

(a)

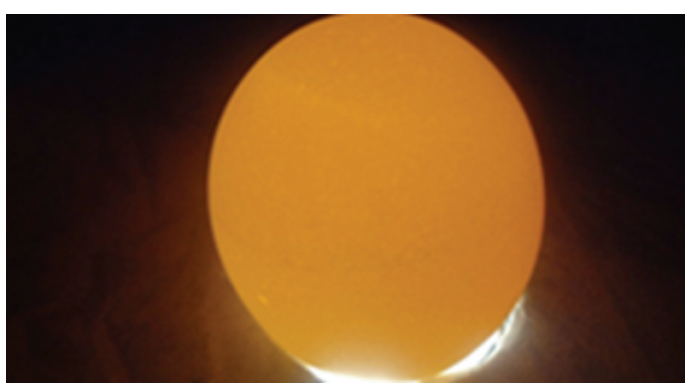

(b)

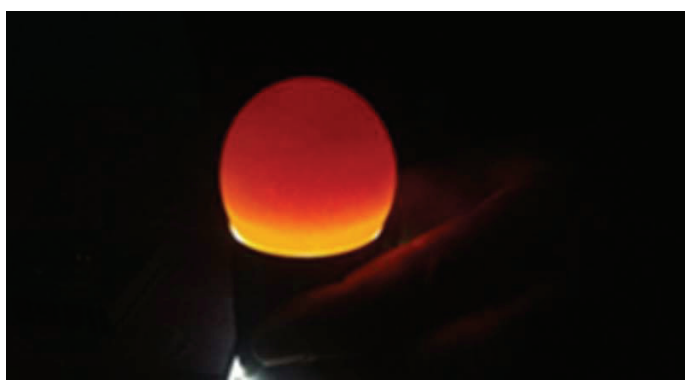

(c)

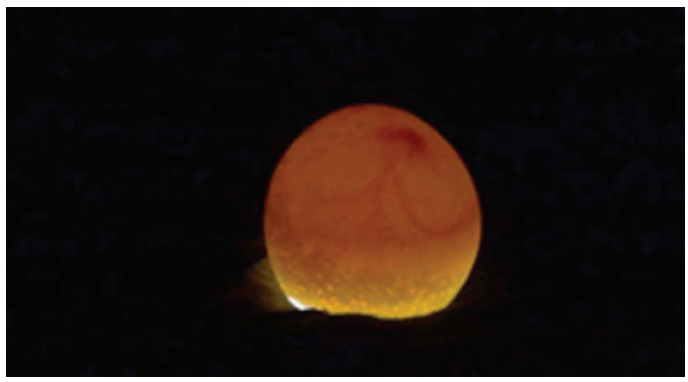

(d)

Fig. 8. Sample images taken by different light sources. (a) Incandescent 200 Watt lamp, (b) Fluorescent lamp, (c) LED 10 Watt, and (d) LED 0.12 Watt.

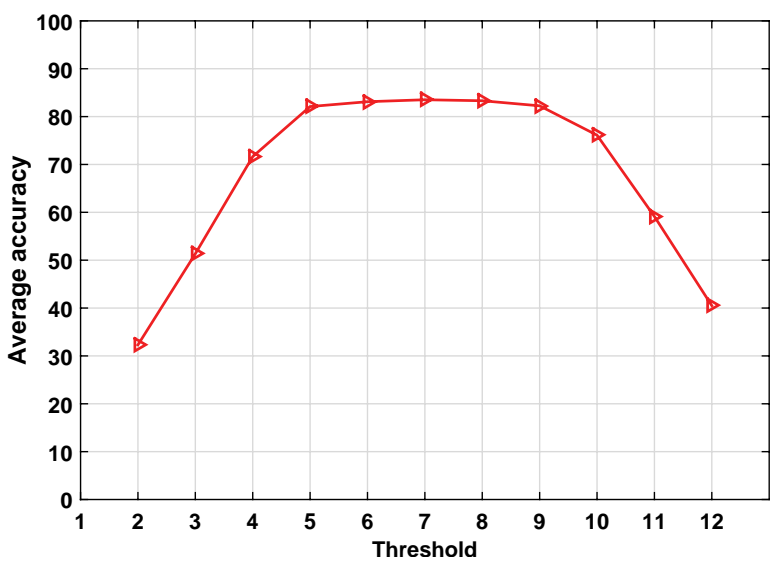

Fig. 9. Average fertility detection accuracies (in percentage) on five days of incubation regarding to the different values of threshold $\beta$.

\section{Conclusion}

In this research, we designed and implemented a very cheap and feasible machine vision system to detect the fertile eggs in the incubation industry. A mechatronic machine was fabricated for acquiring good quality images of eggs without harming them. An appropriate light source was also used for illuminating the eggs, which potentially enabled the system to obtain accurate images from inner side of the eggs. Finally, a robust machine vision algorithm was developed to process the acquired images and distinguish fertile eggs from infertile ones. A large egg image dataset was provided and used to evaluate the proposed system. Average fertility detection rates in the first five days of incubation were $47.13 \%$, $81.41 \%, 93.08 \%, 97.73 \%$, and $98.25 \%$, respectively.

Comparisons with existing approaches showed that the proposed method outperforms more complex approaches. It turns out that the proposed system is highly reliable and applicable in the incubation industry. Despite a few researches done to detect fertilized eggs based on machine vision techniques, our algorithm is simple, yet very efficient in terms of both classification accuracy (e.g. above $97 \%$ at day 4 of incubation) and processing time (less than $0.3 \mathrm{sec}$. per egg). This progress in fertility 
detection can lead to a good profit in the industry, and also prevents damage caused by contaminated eggs inside the incubators. In addition, due to very low power consumption by the imaging light source proposed in this research, the energy usage in this machine is less than the other common candling machines. Moreover, highly accurate images taken by this system can be used in other research related to the incubation industry or any other fields that could be useful.

The proposed machine vision system can be studied and developed from different aspects from which detecting died embryos from other healthy embryos during the incubation process is the most important one. It is better to know that the embryos that die in incubation machines do not have any blood veins and stick to the eggshell, and usually a pink ring, so called blood ring, surrounds it ${ }^{1}$. This fact can be a good starting point for future research in this industry.

\section{References}

1. G. Damerow, Hatching \& Brooding Your Own Chicks: Chickens, Turkeys, Ducks, Geese, Guinea Fowl: Storey Publishing, (2013).

2. L. Liu and M. Ngadi, "Detecting fertility and early embryo development of chicken eggs using nearinfrared hyperspectral imaging," Food and Bioprocess Technology, 6, 2503-2513, (2013).

3. S. Rose, Principles of poultry science: Cab International, (1997).

4. V. Patel, R. McClendon, and J. Goodrum, "Color computer vision and artificial neural networks for the detection of defects in poultry eggs," in Artificial Intelligence for Biology and Agriculture, ed: Springer, 163176, (1998).

5. Y. Usui, K. Nakano, and Y. Motonaga, "A study of the development of non-destructive detection system for abnormal eggs," in EFITA Conference. Debrecen. Hungary, (2003).

6. K. C. Lawrence, S. C. Yoon, G. W. Heitschmidt, D. R. Jones, and B. Park, "Imaging system with modifiedpressure chamber for crack detection in shell eggs," Sensing and Instrumentation for Food Quality and Safety, 2, pp. 116-122, (2008).

7. K. C. Lawrence, S. C. Yoon, D. R. Jones, G. W. Heitschmidt, B. Park, and W. R. Windham, "Modified pressure system for imaging egg cracks," in SPIE Defense and Security Symposium, (2008).

8. X. Deng, Q. Wang, H. Chen, and H. Xie, "Eggshell crack detection using a wavelet-based support vector machine," Computers and Electronics in Agriculture, 70, 135-143, (2010).

9. W. Fang and W. Youxian, "Detecting preserved eggshell crack using machine vision," in Information Technology, Computer Engineering and Management Sciences (ICM), 2011 International Conference on, 62-65, (2011).

10. M. S. Mansoory, M. Ashtiyani, and H. Sarabadani, "Automatic Crack Detection in Eggshell Based on SUSAN Edge Detector Using Fuzzy Thresholding," Modern Applied Science, 5, (2011).

11. R. Ibrahim, Z. M. Zin, N. Nadzri, M. Shamsudin, and M. Zaunidin, "Egg's Grade Classification and Dirt Inspection Using Image Processing Techniques," in Proceedings of the World Congress on Engineering, (2012).

12. Y. Li, S. Dhakal, and Y. Peng, "A machine vision system for identification of micro-crack in egg shell," Journal of Food Engineering, 109, 127-134, (2012).

13. L. Lunadei, L. Ruiz-Garcia, L. Bodria, and R. Guidetti, "Automatic identification of defects on eggshell through a multispectral vision system," Food and Bioprocess Technology, 5, 3042-3050, (2012).

14. V. C. Patel, R. W. McClendon, and J. W. Goodrum, "Development and evaluation of an expert system for egg sorting," Computers and Electronics in Agriculture, 20, 97-116, (1998).

15. K. Mertens, I. Vaesen, J. Lffel, B. Ostyn, B. Kemps, B. Kamers, et al., "Data-based design of an intelligent control chart for the daily monitoring of the average egg weight," Computers and Electronics in Agriculture, 61, 222-232, (2008).

16. P. Javadikia, M. Dehrouyeh, L. Naderloo, H. Rabbani, and A. Lorestani, "Measuring the Weight of Egg with Image Processing and ANFIS Model," in Swarm, Evolutionary, and Memetic Computing. 7076, B. Panigrahi, P. Suganthan, S. Das, and S. Satapathy, Eds., ed: Springer Berlin Heidelberg, 407-416, (2011).

17. K. Brulez, P. Cassey, A. Meeson, I. Mikk, S. L. Webber, A. G. Gosler, et al., "Eggshell spot scoring methods cannot be used as a reliable proxy to determine pigment quantity," Journal of avian biology, 45, 94102, (2014).

18. F. Bamelis, K. Tona, J. De Baerdemaeker, and E. Decuypere, "Detection of early embryonic development in chicken eggs using visible light transmission," British poultry science, 43, 204-212, (2002).

19. K. C. Lawrence, D. P. Smith, W. R. Windham, G. W. Heitschmidt, and B. Park, "Egg embryo development detection with hyperspectral imaging," in Optics East 2006, (2006).

20. D. Smith, K. Lawrence, and G. Heitschmidt, "Fertility and embryo development of broiler hatching eggs evaluated with a hyperspectral imaging and predic- 
tive modeling system," International journal of poultry science, 7, 1001-1004, (2008).

21. C.-S. Lin, P. T. Yeh, D.-C. Chen, Y.-C. Chiou, and C.H. Lee, "The identification and filtering of fertilized eggs with a thermal imaging system," Computers and Electronics in Agriculture, 91, 94-105, (2013).

22. K. Das and M. Evans, "Detecting fertility of hatching eggs using machine vision. I. Histogram characterization method," Transactions of the ASAE (USA), (1992).

23. K. Das and M. Evans, "Detecting fertility of hatching eggs using machine vision. II. Neural network classifiers," Transactions of the ASAE (USA), (1992).

24. Z. Zhu and M. Ma, "The identification of white fertile eggs prior to incubation based on machine vision and least square support vector machine," African Journal of Agricultural Research, 6, 2699-2704, (2011).
25. K. Zuiderveld, "Contrast limited adaptive histogram equalization," in Graphics gems IV, 474-485, (1994).

26. A. M. Tekalp, Digital video processing: Prentice-Hall, Inc., (1995)

27. N. Otsu, "A threshold selection method from graylevel histograms," Automatica, vol. 11, 23-27, (1975).

28. E. Alpaydin, Introduction to machine learning: MIT press, (2014)

29. L. Fausett, Fundamentals of neural networks: architectures, algorithms, and applications: Prentice-Hall, Inc., (1994).

30. G. Bradski and A. Kaehler, Learning OpenCV: Computer vision with the OpenCV library, O'Reilly Media, Inc., (2008)

31. OpenCV. (2014). Available: http://sourceforge. net/projects/opencvlibrary/ 